\title{
BMJ Open Quality Time for change in practice of in-patient oxygen therapy: a period-limited, multidimensional approach to improve oxygen prescription compliance: quality improvement project at Hamad General Hospital, Qatar
}

Samman Rose, Sundus Sardar (D), Sreethish Sasi, Dabia Hamad S H Al Mohanadi, Ahmed Ali A A Al-Mohammed, Muhammad Zahid

To cite: Rose S, Sardar S, Sasi S, et al. Time for change in practice of in-patient oxygen therapy: a periodlimited, multidimensional approach to improve oxygen prescription compliance: quality improvement project at Hamad General Hospital, Qatar. BMJ Open Quality 2021;10:e001574. doi:10.1136/ bmjoq-2021-001574

Received 4 June 2021 Accepted 21 October 2021

Check for updates

(c) Author(s) (or their employer(s)) 2021. Re-use permitted under CC BY-NC. No commercial re-use. See rights and permissions. Published by BMJ.

Internal Medicine, Hamad General Hospital, Hamad Medical Corporation, Doha, Qatar

Correspondence to Dr Samman Rose; dr.sam.rose@gmail.com

\section{ABSTRACT}

Prescription of oxygen therapy has traditionally poor compliance across the globe and mostly given to patients on verbal orders leading to under or overuse. The British Thoracic Society (BTS) guidelines (2017) recommend that oxygen therapy must be prescribed. Our study aimed to assess the prescription practice of oxygen therapy for patients admitted to acute medical assessment unit and general medical wards at Hamad General Hospital, Qatar and to achieve $80 \%$ compliance of valid oxygen therapy prescription implementing the quality improvement model against the BTS guidelines.

The prescription practice of oxygen therapy was audited between April 2019 and August 2019. Using a Plan, Do, Study, Act (PDSA) model of improvement and multiple interventions was performed in the eight PDSA cycles, including (1) educational sessions for residents/fellows/ nurses, (2) introduction of electronic prescription, (3) emails, posters/flyers, (4) nurse-led reminders and (5) re-enforced teaching for new residents. Data were then collected using a questionnaire assessing electronic prescriptions and documentation. Our baseline study regarding oxygen therapy showed limited awareness of BTS guidelines regarding the documentation of initiation and further adjustment of oxygen therapy. There was a lack of compliance with oxygen prescription; none of the patients had a valid prescription on our computerbased prescription (Cerner). The duration, target range and indications of Oxygen therapy were documented in $25 \%$ (18/72), 45.8\% (33/72) and 42\% (30/72) patients, respectively. 0xygen was initiated by communication order only. In a total of 16 weeks period, the repeated PDSA cycles showed significant improvement in safe oxygen prescription practices. Following intervention, oxygen electronic prescription, documentation of indications for oxygen therapy, target oxygen saturation and wean-off plan improved to $93 \%, 85 \%, 86 \%$ and 80 $\%$, respectively.

We concluded that poor compliance to oxygen therapy Orders is a universal issue, which can be successfully managed using small-scale PDSA cycles to ensure sustained improvement through multidimensional interventions, continuous reinforcement and frequent reassessments.

\section{PROBLEM}

Oxygen, one of the most commonly used drugs, is seldom prescribed/ordered in medical records. An audit of in-patient hospital patients by The BTS 2015 showed that those on oxygen therapy had no valid orders for $42.5 \%$. ${ }^{1}$ A recent study by Chu et al demonstrated that liberal oxygen therapy is associated with high mortality, and therefore, oxygen should be given within a specific range determined at the time of prescription based on underlying disease processes. ${ }^{2}$ Excessive and uncontrolled oxygen therapy can lead to hypercapnia in patients with chronic obstructive pulmonary disease (COPD). ${ }^{3}$ In recent times, several international bodies have advocated for the prescription of oxygen therapy to reduce the risk in vulnerable patient groups. ${ }^{4}$ Despite this guidance, published data have demonstrated that there has been poor uptake of these recommendations. This has led to higher levels of oxygen therapy being delivered to patients who are critically unwell or who have dyspnoea, ${ }^{5}$ resulting in increased length of stay, higher rates of admission to high dependency units ${ }^{6}$ and an increased risk of death. ${ }^{78}$

In our project, we define prescription of oxygen therapy as an electronic order comprising of the components: indication, mode, rate, duration and wean off planautomatically available for nursing and allied staff to follow instructions with specified date and time. We used the We assessed the prescription of oxygen categorically in the following main areas: (1) compliance with 
oxygen prescription, and (2) awareness of BTS guidelines for its use. The majority of the admitted patients who were started on oxygen therapy had no documentation in the electronic medical record (EMR)/Cerner system of its prescription (indication, target, route of delivery).

Our study aimed to assess the prescription (electronic orders) practice of oxygen therapy for patients admitted to acute medical assessment unit and general medical wards at Hamad General Hospital, Qatar, and to achieve $80 \%$ compliance of valid oxygen therapy prescription, implementing the quality improvement model against the BTS guidelines.

\section{BACKGROUND}

The BTS guideline states that all patients requiring Oxygen must have valid orders on admission and target saturations must be stipulated on the prescription chart. ${ }^{9}$ Jeffrey et $a l^{10}$ demonstrated that only $43 \%$ of patients on supplemental Oxygen received the prescribed therapy.

An audit (Neill et $a l^{11}$ ) at performed at a Christchurch Newzeland hospital showed an assessment and management of patients admitted with COPD. The study identified oxygen prescription issues in in addition to other deficiencies. Oxygen therapy was required in $87 \%$ of cases while One third had no oxygen prescription or adequate monitoring of oxygen therapy documented.

Based on BTS guidelines, ${ }^{9}$ the orders should include the starting oxygen dose and oxygen delivery device, and most importantly, the oxygen saturation target range; similarly, any change must be documented. A quality improvement project/audit of oxygen-therapy at UK hospitals by O'Driscoll $e^{\prime} \mathrm{al}^{12}$ during the period of 2008-2013 revealed that prescriptions with a target saturation range written increased from $10 \%$ in 2008 to $52 \%$ in 2013 , while $45 \%$ of patients on oxygen therapy had no written orders.

From the available literature, it is clear that oxygen therapy prescription/orders is a universal issue, and several audits and Quality improvement projects have been performed to identify the areas of non-compliance and improve the prescription safely. We in our study found similar compliance issue as prevalent across the globe. In a study, ${ }^{13}$ the compliance of Oxygen orders rates increased to $69 \%$ following tailored interventions for specific healthcare professional groups, including the junior doctors (prescribers) and Nursing staff (administrators of Oxygen), by raising awareness in the nursing staff and introduction of a bedside warnings for the patients receiving oxygen therapy. Similarly, in other studies, the areas of non-compliance, particularly the lack of awareness regarding the importance of Oxygen as a 'drug', were addressed by educating prescribers, including doctors and nurses, through education and other targeted interventions. ${ }^{1415}$

\section{Current practice of oxygen prescription}

Initially, the general practice of oxygen prescription/ orders is via communication orders, mostly incomplete without clear indication, mode, target saturation or wean-off plan. The communication order is essentially an informal directive entered into the EMR/Cerner system delineating general instructions from physicians to nurses regarding patient care and management plans, such as 'please administer oxygen $2 \mathrm{~L} / \mathrm{min}$ '. We introduced the use of official oxygen therapy orders to clearly document indication, mode, target saturation and wean-off plan for oxygen prescription. The fishbone diagram (figure 1) depicts the issues encountered with oxygen therapy prescription.

\section{Measurement}

An initial baseline study was conducted among inpatients of acute medical assessment unit and medical wards requiring oxygen treatment during March 2019, evaluating the compliance to oxygen prescription guidelines.

EMRs (Cerner) were used to collect the information. Data included a review of oxygen prescription order on EMR/Cerner to review the documentation including electronic prescription, indication for oxygen documentation, oxygen saturation with target $\%$, the percentage of patients within that target range and wean off plans in admission and daily progress notes for every patient requiring oxygen therapy. Staff, including doctors and nurses, was surveyed to evaluate their awareness of BTS guidelines regarding oxygen use. Data were recorded on a Microsoft Excel sheet.

\section{BASELINE DATA}

\section{Patient characteristics}

A total of 72 patients were included in the baseline audit.

- Mean age was $62 \pm 18$ years (median - 67years).

- $49 \%$ were male $(35 / 72), 51 \%$ were female $(37 / 72)$.

- None of the patients had a valid prescription on CERNER (electronic database).

- The duration, target range and indications for oxygen therapy were documented through communication orders in $25 \%(18 / 72), 45.8 \%(33 / 72)$ and $42 \%$ $30 / 72$ patients, respectively.

- Data collection was time period bound, meaning all patients admitted to acute medical admission unit (AMAU) requiring oxygen therapy in a 4 weeks time.

- All patients were non-critically ill admitted to AMAU or the medical floor.

Our baseline study shows that there is limited awareness of BTS guidelines in the following aspects:

- Initiation of oxygen therapy-rate, route and type.

- Target saturation specific about each condition.

- Weaning-off oxygen therapy.

Our initial audit survey results showed that none of the patients had appropriate oxygen therapy orders as per standard.

- A total of $75 \%$ of patients had oxygen prescribed as only communication order, and $25 \%$ had no orders. 


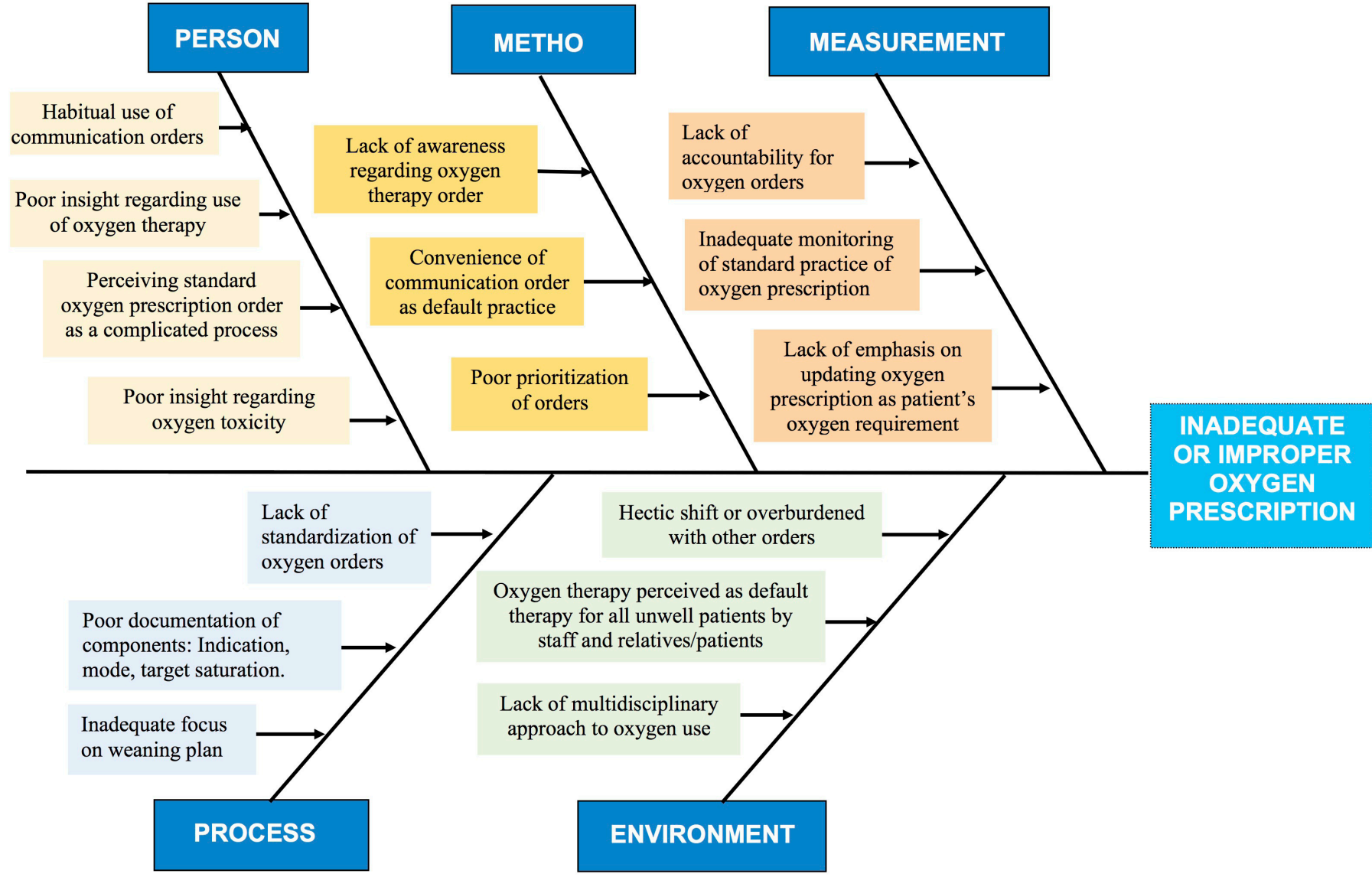

Figure 1 The fishbone diagram identifying and analysing potential contributing root causes for inadequate or improper oxygen prescription.

- A total of $47 \%$ of the patients on oxygen therapy had their oxygen saturations out of their recommended range.

- A total of $55 \%$ of the patients had no indication for oxygen therapy documented in their notes.

\section{METHODS}

The prescription/orders practice of oxygen therapy was audited between April 2019 and August 2019. The current practice of junior doctors/Nurses was assessed using a designed proforma against the standard BTS guidelines. The data were collected using CERNER (database) from admitted patients in an acute medical assessment unit and general medical wards. Standard statistical methods, including mean, median and SD, were used to analyse the data.

A Survey was conducted to judge doctors and nurses' practice and perception regarding oxygen therapy. The survey of 50 doctors prior to start of the project showed $8 \%$ were aware of oxygen prescription policy of the hospital, $12 \%$ did not considered oxygen as medicine and $18 \%$ did not think oxygen should be prescribed.

Our Quality Improvement Programme team comprised six junior residents and a team leader, all part of the internal medicine residency programme. Initial Interventions were implemented before the first PDSA cycle based on initial baseline data and after discussion among team members and nursing staff of the assigned medical areas. Results from each PDSA cycle were studied, and regular meetings among team members' interventions were introduced at each step accordingly.

Interventions were decided based on baseline data, and a doctors and nurses survey was done to assess their awareness of BTS guidelines regarding oxygen use and valid oxygen therapy orders.

Interventions included junior doctors' education about the Importance of safe oxygen therapy and how to place an oxygen prescription/order on EMR/Cerner, including Mode of oxygen, target saturation, indication for oxygen and wean-off plan. They were also encouraged to document indication and wean-off plan daily in their progress notes for every patient requiring oxygen therapy. Nurses were requested to follow oxygen prescriptions and remind the physicians if there were none. Multiple interventions were introduced systematically during each cycle/PDSA cycle (A) lectures for residents/fellows/ nurses (B) communication via emails and group SMS/ Whatsapp (C) posters and flyers near resident lounges, nursing stations and patient beds (E) daily prompting of the multidisciplinary teams by a member of the project team (F) re-enforced teaching for newly joined junior doctors and (G) nurse-led reminders. Data were collected 
using a questionnaire assessing electronic prescriptions and documentation.

\section{STRATEGY}

Eight PDSA cycles were completed over 16 weeks from April 2019 to August 2019. In each cycle, an intervention was implemented and studied for 2 weeks. Results were analysed, and feedback was taken from multidisciplinary teams. Further interventions were explored in subsequent PDSA cycles along with reinforcement of the previous one.

\section{PDSA CYCLE}

\section{PDSA cycle 1}

All PDSA cycles were conducted on the same designated medical teams. In the first PDSA cycle (sample size: 23 patients), introductory lectures were arranged as an intervention at a departmental level during the morning educational activities. Here, we introduced the quality improvement project, educated junior doctors regarding BTS guidelines for oxygen prescription and highlighted the components for oxygen prescription as per international guidelines.

We identified the factors that may hinder the project and the appropriate orders of oxygen therapy. The most important factor to highlight is that many physicians believe that oxygen is not a drug and does not require a prescription. Our introductory lectures helped to emphasise the adverse effects of inappropriate use of oxygen therapy and convey the importance of appropriate oxygen prescription/orders. Along with emphasising oxygen as a drug, we also stressed the importance of oxygen saturation targets designated for specific medical conditions.

Compliance with the proper prescription guidelines was $34 \%$, with $56 \%$ documentation indicating oxygen therapy, and 53\% documented a wean-off plan. Only $62 \%$ of patients had oxygen saturations recorded in target ranges as recommended by guidelines.

\section{Lesson learnt}

Launching with the introductory lecture helped to challenge the common misperception that oxygen is not a drug and emphasise the basic concept that oxygen therapy requires a prescription focusing on the indication, target saturation and wean-off plan.

\section{PDSA cycle 2}

This cycle involved disseminating posters and flyers within the patient wards. The poster elaborated information regarding indication, oxygen targets, wean-off plans. It also illustrated the process of electronic oxygen prescription via a pictorial diagram depicting all the oxygen therapy prescription steps on the electronic medical viewer system. These posters were distributed in doctors' and nurses' working stations and residents' recreational lounges.
In PDSA cycle 2 (sample size=25), compliance with the proper prescription guidelines was $45 \%$ with $65 \%$ documentation for oxygen therapy indication, and $64 \%$ documented a wean-off plan. $65 \%$ of patients had oxygen saturations recorded in target ranges as recommended by guidelines.

\section{Lessons learnt}

Visual reminders of the importance of oxygen therapy prescription in the medical wards improved appropriate electronic prescription of oxygen therapy.

\section{PDSA cycle 3}

The intervention for this PDSA cycle (sample size: 24) focused on educating the nurses regarding appropriate oxygen orders required from physicians. We held wardbased small teaching lectures targeted for the nurses to emphasise the importance of a valid oxygen prescription. In these lectures, we stressed recommendations for appropriate oxygen saturation targets in various medical conditions. We also highlighted that it is essential for all healthcare professionals to play their part to ensure appropriate electronic oxygen prescription. After the lectures, we assigned designated nurses for each ward to lead the physician reminders for oxygen therapy orders.

Compliance with the proper prescription was $57 \%$ with $75 \%$ documentation for oxygen therapy indication, and $68 \%$ documented a wean-off plan. $70 \%$ of patients had oxygen saturations recorded in target ranges as recommended by guidelines.

\section{Lessons learnt}

Empowering nurses by involving them in this cycle helped educating them about appropriate oxygen therapy, emphasise that oxygen is a drug and should be prescribed and weaned off on time. Nurse-led reminders improved compliance to write appropriate oxygen therapy prescriptions with a focus on indication and wean-off plans. Incorporating guidelines to emphasise appropriate target oxygen saturations also improved the number of patients with oxygen saturations recorded within the recommended target range.

\section{PDSA cycle 4}

Given the previous PDSA cycle results, we launched emails and messages to send official reminders to all ten General Medicine teams, including resident physicians, medical students and consultants. The emails included reminders for oxygen prescription, links to BTS guidelines, and the pictogram instruction to electronic input prescription for oxygen therapy. These emails were sent system-wide to ensure all physicians in General Medicine teams are well informed regarding appropriate oxygen orders. It also emphasised continuing the chain of education in their respective groups and medical teams. We also added reminder badges for the nurses and physicians. These were placed onto each computer in the medical wards to ensure a visual reminder for oxygen therapy orders. 
In PDSA cycle 4 (sample size: 22), compliance with proper prescription guidelines was $57 \%$ with $75 \%$ documentation for oxygen therapy indication, and $68 \%$ documented a wean-off plan. $70 \%$ of patients had oxygen saturations recorded in target ranges as recommended by guidelines.

\section{Lesson learnt}

Here, we approached the system to ensure each member takes responsibility and emphasises that consultants supervise their junior doctors to implement appropriate oxygen therapy prescription system-wide.

\section{PDSA cycle 5}

In this cycle (sample size: 21), we reinforced all previous interventions conducted until now. As the last cycle coincided with new resident physicians' intake, we arranged for junior doctor orientation sessions, incorporating appropriate oxygen prescription training. We also circulated flow chart prescription cards illustrating valid electronic orders for oxygen therapy.

Compliance with the proper prescription guidelines was $65 \%$ with $80 \%$ documentation for oxygen therapy indication, and $70 \%$ documented a wean-off plan. Sixty-six per cent of patients had oxygen saturations recorded in target ranges as recommended by guidelines.

\section{Lesson learnt}

While implementing visual reminders helped the previous batch of residents, these measures alone were not enough to orient the incoming junior residents. We took quick action to incorporate orientation sessions for the new residents and emphasised the importance of an appropriate electronic prescription for oxygen therapy with a clear indication, wean-off plan, and target saturation.

\section{PDSA cycle 6}

In PDSA cycle 6 (sample size: 19), we selected resident physicians as 'oxygen warrior' for the designated medical teams. One junior doctor from each of the ten medical teams was chosen for the oxygen warrior's role in leading among their teams and ensuring all patients admitted to their team have appropriate oxygen therapy orders and valid oxygen prescriptions with all the necessary components. These residents also reviewed their team's patient files every 24 hours to ensure all patients have electronic prescriptions for oxygen therapy with appropriate wean-off oxygen plans, which were discussed daily with consultants and patients.

Compliance with the proper prescription guidelines was $70 \%$ with $80 \%$ documentation for oxygen therapy indication, and $75 \%$ documented a wean-off plan. Seventy-seven per cent of patients had oxygen saturations recorded in target ranges as recommended by guidelines.

\section{Lessons learnt}

The designated residents in the active role of 'oxygen warriors' gained a sense of responsibility, good knowledge of patient history as required for appropriate oxygen orders. Introducing 'oxygen warrior' also encouraged residents to develop good communication among team members and consultants regarding the daily discussion about oxygen wean-off plans and facilitate patient education regarding oxygen use.

\section{PDSA cycle 7}

Here, we conducted one-to-one teaching for residents to reinforce appropriate administration of oxygen therapy. In this cycle (sample size: 31), the number of prescriptions for oxygen therapy was improving. We obtained feedback from residents and looked into any barriers hindering appropriate oxygen therapy prescription. We also identified if anyone was not confident with oxygen prescription, we performed one-to-one teaching. For the residents who exhibited proper oxygen therapy prescriptions in line with the guidelines, we provided positive feedback to the teams and consultants to encourage these team members.

Compliance with the proper prescription guidelines was $77 \%$ with $84 \%$ documentation for oxygen therapy indication, and $78 \%$ documented a wean-off plan. A total of $88 \%$ of patients had oxygen saturations recorded in target ranges as recommended by guidelines.

\section{Lessons learnt}

Here, we analysed the fishbone diagram in detail to ascertain barriers to appropriate oxygen prescription. One of the factors hindering electronic prescription was that residents deemed it time-consuming amidst routine physician duties; thus, we explained the process with pictograms and arranged education regarding the main components of a valid prescription.

\section{PDSA cycle 8}

In the last PDSA cycle, we identified any further deficits and reinforced all previous interventions. We monitored all ten medical teams, requested oxygen warriors for feedback regarding any noted shortfalls. It was determined that some residents had issues with indications and wean-off plans, and thereby we encouraged residents and medical students to approach consultants during ward rounds to discuss the wean-off plans and thoughtfully approach the indication for therapy.

In PDSA cycle 8 (sample size: 24), compliance with the proper prescription guideline was $81 \%$ with $85 \%$ documentation for oxygen therapy indication, and $80 \%$ documented a wean-off plan. $93 \%$ of patients had oxygen saturations recorded in target ranges as recommended by guidelines.

\section{Lessons learnt}

In this cycle, we noted that designating a platform to openly discuss any difficulties and deficits led to bettertargeted instruction regarding the electronic prescription contents for oxygen therapy. It also encouraged physician awareness regarding appropriate oxygen therapy and patient education, thereby improving patient care, 


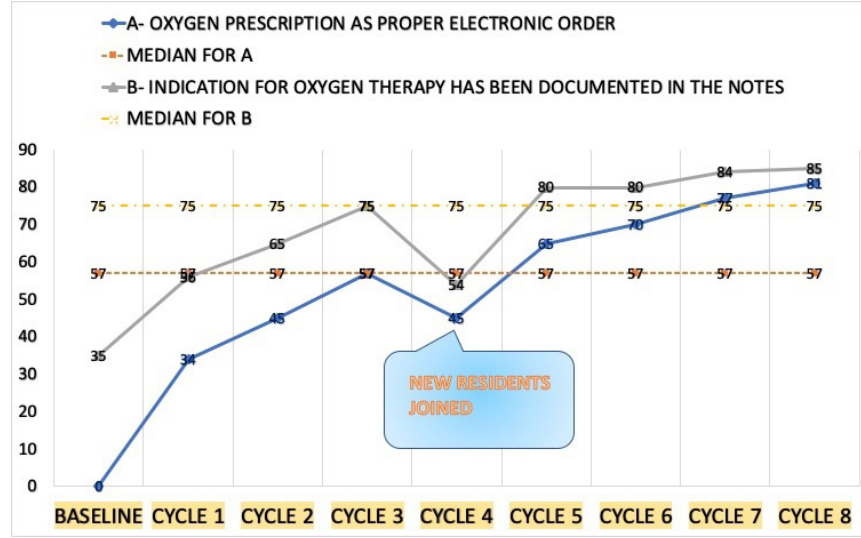

Figure 2 Run chart demonstrating oxygen prescription indication and as an electronic order in notes.

patient education and awareness regarding appropriate oxygen prescription.

Overall, we created a nurse-incorporated physicianchampioned system for oxygen therapy prescriptions, ensuring good compliance to use recommendations as per guidelines.

\section{RESULTS}

The data were recorded in a Microsoft Excel spreadsheet. Run charts were prepared for each category with a target median and achieved a median (figures 2 and 3).

- Process measures: Patients with oxygen prescriptions on EMR improved to $81 \%$ (target $70 \%$ ) . Documentation regarding oxygen therapy indication, target saturation and wean-off plan improved to $85 \%, 86 \%$ and $80 \%$, respectively.

- Outcome measure: Percentage of patients with oxygen saturation in the target range improved from $41 \%$ at baseline to $93 \%$, signifying lesser adverse effects of over or under oxygenation.

- Balancing measure: Increased awareness of junior doctors, nurses, and allied staff in the safe use of therapeutic oxygen.

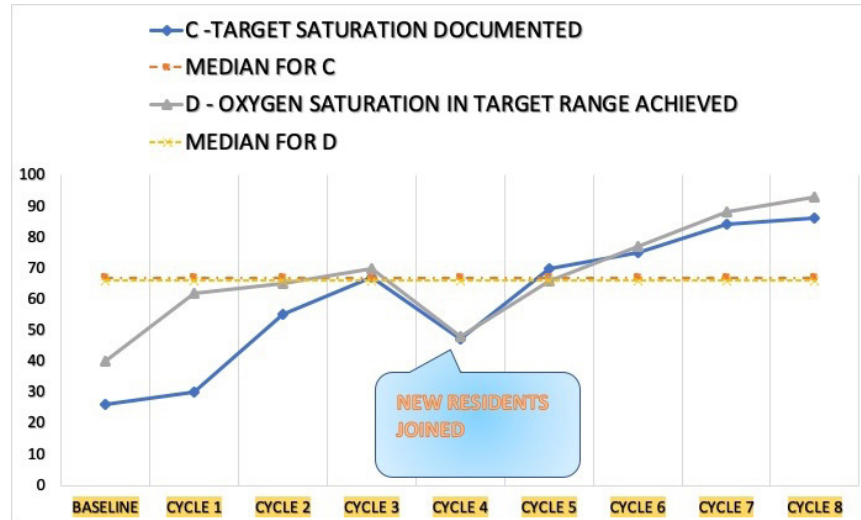

Figure 3 Run chart demonstrating documentation of target saturation and target range achieved.

\section{Effects of changes}

In 16 weeks of the intervention period, repeated PDSA cycles showed significant improvement in safe oxygen orders practices, as shown in charts (figures 2 and 3 ).

\section{LESSONS AND LIMITATIONS}

The interventions in our quality project addressed multiple aspects including educational interventions, reminders and EMR system-based modifications. Interventions comprised of monthly reminders on secured medical team communication groups, nurse-led reminders as part of admission bundle and reinforcing initiation of oxygen therapy based on standardised electronic prescription with all indicated components, and EMR system popups and autoreminders.

To keep standardised practice in our hospital—we have a regular auditing team which regularly monitor quality as part of Joint commission international programme, a global leader for healthcare quality accreditation. Any non-compliance is identified and corrected. Until recently our oxygen therapy orders has higher range of compliance.

Some limitations and challenges included:

- Rotation of junior doctors in each medical team every 4 weeks is the biggest hurdle to sustain the change.

- The permanent staff of the unit, that is, team consultant, nurses and clinical pharmacists, are vital members to sustain the change and play an essential role in training and teaching junior doctors rotating in medical teams.

- Continuous effort in the form of a multidisciplinary approach, teaching and training of staff and periodic reinforcement is necessary to bring about sustainable change.

- The recommendations from the study are an ongoing process at our institute well beyond 16 weeks. It is still checked as a part of our hospital policy.

\section{CONCLUSIONS}

Although oxygen is a frequently used drug, its prescription/orders remains under-utilised. Our project's main aim focused on enhancing physicians and nurses' knowledge to achieve $80 \%$ compliance with appropriate oxygen prescription. We emphasised a multidisciplinary approach with multidimensional intervention to achieve and maintain the set targets-this project utilised visual reminders and continuous educational sessions to sustain and re-enforce interventions. One of the challenges was monthly rotations of resident physicians; thus, regular sessions were organised to re-emphasise interventions. Implementing an appropriate oxygen prescription with vital three components stressed on the essential factors of oxygen use. Therefore, physicians were able to incorporate electronic oxygen prescription into their clinical practice effectively.

We are developing 1-hour multidisciplinary oxygen prescribing/orders and delivery lecture for our clinical 
ward staff. We recommend that oxygen prescription training be incorporated as an essential component of the orientation sessions for newly-joining physicians. Further PDSA cycles are planned to assess future compliance and attain sustainability to appropriate oxygen prescription. We have updated our local hospital policy on oxygen use and designing an e-learning module for our clinical staff. In future, we are planning to educate patients on oxygen use and its side effects. We will involve them in the target setting and wean-off plan.

Acknowledgements The authors would like to thank Hamad General Hospital leadership including Dr Dabia Hamad S H Al Mohanadi and Dr Ahmed Ali A A Al-Mohammed for their support to our project. The authors appreciate Yazan Almohtasib, Urshita Sinha, and Reham Abo Shdid for their contributions to the project. The authors would especially like to thank the nursing staff and the Internal Medicine Residency Programme for their kind assistance to the initiative.

Contributors SR and MZ conceptualised, designed and supervised the project. SR, SSar and SSas prepared the initial draft of this manuscript. SR, SSar and SSas acknowledged team members contributed in testing and implementation of change ideas, data collection, PDSA cycles and analysis of data. SR, SSar and MZ revised the manuscript critically and conducted the relevant literature review.SR is the guarantor and accepts full responsibility for the finished work and/or the conduct of the study, had access to the data, and controlled the decision to publish.

Funding The authors have not declared a specific grant for this research from any funding agency in the public, commercial or not-for-profit sectors.

Competing interests None declared.

Patient and public involvement Patients and/or the public were not involved in the design, or conduct, or reporting, or dissemination plans of this research.

Patient consent for publication Not required.

Ethics approval The project was exampted from ethical approval as this was a quality improvment project. Any further institutional review board approval not required as the changes being tested and implemented are evidence-based and widely accepted internationally. The project did not include any control group or randomisation; therefore, no patients would be denied the best intervention.

Provenance and peer review Not commissioned; externally peer reviewed. Data availability statement Data are available upon request.

Open access This is an open access article distributed in accordance with the Creative Commons Attribution Non Commercial (CC BY-NC 4.0) license, which permits others to distribute, remix, adapt, build upon this work non-commercially, and license their derivative works on different terms, provided the original work is properly cited, appropriate credit is given, any changes made indicated, and the use is non-commercial. See: http://creativecommons.org/licenses/by-nc/4.0/.
ORCID iD

Sundus Sardar http://orcid.org/0000-0002-1727-0520

\section{REFERENCES}

1 O'Driscoll R. British thoracic Society emergency oxygen audit report. BTS national respiratory audit programme. annual report, 2015. Available: https://www.brit-thoracic.org.uk/document-library/auditand-qualityimprovement/audit-reports/bts-emergency-oxygen-auditreport-2015/ [Accessed 30 Dec 2020].

2 Chu DK, Kim LH-Y, Young PJ, et al. Mortality and morbidity in acutely ill adults treated with liberal versus conservative oxygen therapy (iota): a systematic review and meta-analysis. Lancet 2018;391:1693-705.

3 Plant PK, Owen JL, Elliott MW. One year period prevalence study of respiratory acidosis in acute exacerbations of COPD: implications for the provision of non-invasive ventilation and oxygen administration. Thorax 2000;55:550-4.

4 Cousins JL, Wark PAB, McDonald VM. Acute oxygen therapy: a review of prescribing and delivery practices. Int J Chron Obstruct Pulmon Dis 2016;11:1067-75.

5 Kane B, Decalmer S, Ronan O'Driscoll B, O’Driscoll BR. Emergency oxygen therapy: from guideline to implementation. Breathe 2013;9:246-53.

6 Joosten SA, Koh MS, Bu X, et al. The effects of oxygen therapy in patients presenting to an emergency department with exacerbation of chronic obstructive pulmonary disease. Med J Aust 2007; 186:235-8.

7 Austin MA, Wills KE, Blizzard L, et al. Effect of high flow oxygen on mortality in chronic obstructive pulmonary disease patients in prehospital setting: randomised controlled trial. BMJ 2010;341:c5462.

8 Wijesinghe M, Perrin K, Healy B, et al. Pre-Hospital oxygen therapy in acute exacerbations of chronic obstructive pulmonary disease. Intern Med J 2011;41:618-22.

9 O'Driscoll BR, Howard LS, Earis J. Bts guideline for emergency oxygen use in adult patients. Thorax 2017;72.

10 Jeffrey AA, Ray S, Douglas NJ. Accuracy of inpatient oxygen administration. Thorax 1989;44:1036-7.

11 Neill AM, Epton MJ, Martin IR, et al. An audit of the assessment and management of patients admitted to Christchurch hospital with chronic obstructive pulmonary disease. N Z Med J 1994;107:365-7.

12 O'Driscoll BR, Howard LS, Bucknall C, et al. British thoracic Society emergency oxygen audits. Thorax 2011;66:734-5.

13 Helliar S. Improving oxygen prescribing rates by tailoring interventions for specific healthcare professional groups. BMJ Qual Improv Rep 2016;5. doi:10.1136/bmjquality.u209520.w4033. [Epub ahead of print: 1312 2016].

14 Gatter M, Dixon G, Wall J, et al. Changing an ingrained culture: improving the safety of oxygen therapy at university hospitals Bristol NHS Foundation trust. BMJ Qual Improv Rep 2015;4. doi:10.1136/ bmjquality.u203238.w1474. [Epub ahead of print: 2407 2015].

15 Dimock RAC, Stuart S, Padmanaban V, et al. P64 The impact of simple interventions on oxygen prescribing and monitoring: Audit of oxygen management in Central London Teaching Hospital. Thorax 2013;68:A103::2-104. 